\title{
Buoyancy effects on objects moving in a bubbling fluidized bed
}

\author{
A. Soria-Verdugo*, L.M. Garcia-Gutierrez, N. García-Hernando, U. Ruiz-Rivas \\ Carlos III University of Madrid, Energy Systems Engineering Group, Thermal and Fluids Engineering Department, Avda. de la Universidad 30, 28911 Leganés, Madrid, Spain
}

Keywords:

Gas fluidization

Bubbling bed

Object motion

Buoyancy effect

Rising and sinking path

Number of jumps

\begin{abstract}
A B S T R A T
The effect of buoyant forces on the motion of a large object immersed in a bubbling fluidized bed (BFB) was experimentally studied using digital image analysis. The experiments were performed in a 2 D bubbling fluidized bed with glass spheres as bed material and cylindrical objects with different densities and sizes. The object motion was measured using non intrusive tracking techniques. The effect of gas velocity was also analyzed.

The circulation of an object in a BFB is defined by several parameters. The object might be able to circulate homogeneously throughout the bed or stay in preferred regions, such as the splash zone or the bottom zone. While circulating, the object moves back and forth between the surface of the bed and the inner regions, performing a series of cycles. Each cycle is composed by sinking and rising paths, which can be one or several, depending on whether a passing bubble is able to lift the object to the surface or the object is detached from it or its drift at an intermediate depth. Therefore, the number of rising paths or number of jumps that the object undergo in a cycle, interleaved with sinking paths, and the maximum attained depth characterize each cycle, together with the mean sinking and rising velocities of the object. In this work, experimental measurements of the probability distributions of the number of jumps and the maximum attained depth, the axial homogeneity of object motion and rising and sinking object velocities are presented for objects with different sizes and densities. The results show a coherent behavior, independent of density and size, for the probability distributions of the number of jumps. This is also true for the maximum attained depth, but only when a proper circulation throughout the bed is ensured. Such a proper circulation and axial homogeneity is, on the other hand, much affected by object density, size and gas velocity. Rising and sinking velocities are highly dependent on gas velocity, as established in well known models of bubble and dense phase velocities. Nevertheless, rising velocities are practically unaffected by object density or size, while sinking velocities show a low dependence on density and a steeper one on size. These results suggest that buoyant forces are relevant during the sinking process, and almost neutral during the rising path.
\end{abstract}

\section{Introduction}

Fluidized beds are employed in industry because of their excellent properties involving heat and mass transfer, and their capability to establish and promote chemical reactions inside them. Several processes can occur inside a fluidized bed, such as drying, thermal conversion of solid fuels and coating of particles. In parallel to the wide range of applications, there is a wide range of scales concerning fluidized beds, from small dryers to huge fluidized bed reactors. Most of these applications involve the motion of objects inside the bed. Fuel particles, catalysts and agglomerates are examples of typical objects found inside a fluidized bed. It is necessary to characterize the motion of these objects within the bed to prevent operational problems such as

\footnotetext{
* Corresponding author. Tel.: + 34916248884; fax: + 34916249430 .

E-mail address: asoria@ing.uc3m.es (A. Soria-Verdugo).
}

the existence of hot or cold spots and the appearance of de fluidized zones due to the existence of agglomerates.

Different experimental techniques have been used to describe the motion of tracer particles both in $2 \mathrm{D}$ and $3 \mathrm{D}$ beds. Concern ing $3 \mathrm{D}$ beds, tomography tracking techniques such as positron emission (Stein et al., 2000), X ray (Grassler and Wirth, 2000) and electrical capacitance (Du et al., 2002) methods are common. Nevertheless non tracking techniques have also been employed to collect data of the motion at a particular position in the bed (Ibsen et al., 2002). On the other hand, when analyzing objects motion in a 2 D bed, visualization techniques (Shen et al., 2004; Bokkers et al., 2004) have been employed.

Kunii and Levenspiel (1991) reviewed the first studies intended to characterize the motion of large objects immersed in a fluidized bed. It is generally accepted that objects sink in the bed with the dense phase and rise by the action of ascending bubbles. The motion of large objects in both $2 \mathrm{D}$ and $3 \mathrm{D}$ beds was analyzed by Rios et al. (1986), focusing on the sinking and 
rising processes. They reported that the mean rising velocity of objects was lower than the velocity of bubbles due to the fact that an object generally needs to be lifted by a number of passing bubbles to rise to the freeboard, conforming a rising path composed of multiple jumps that they called jerks. Pallarès and Johnsson (2006) tracked a phosphorescent particle moving in a 2 D bed operating with different bed parameters: air velocities and bed heights, giving a varying amount of vortex structures. In a 2 vortex configuration, a configuration found in most cases with the exception of very shallow beds, they found a general tendency of objects to sink at the sides of the bed and rise at the center, which is generally accepted. Lim and Agarwal (1994) found that a neutrally buoyant object circulated in a 2 D bed throughout the whole bed, with a sinking velocity which was in good agreement with the Kunii and Levenspiel (1991) correlation for the velocity of the dense phase. The evaluation of the mean rising velocity of the object and its relation with the bubbles velocity is a more complex task, due to the multiple jump behavior observed by Rios et al. (1986). Nonetheless, the experimental works of Nienow et al. (1978), Lim and Agarwal (1994) and Rees et al. (2005) define a rough estimate that varies between authors and sets the mean rising velocity of objects between $10 \%$ and $30 \%$ of the mean bubble velocity along the bed.

The effect of buoyant forces has been also the focus of several works. Tanimoto et al. (1981) reported a relative motion between an object and the dense phase. The relative velocity was linear with density, for large density ratios. Nevertheless, the buoyant force suffered by an object immersed in a bubbling fluidized bed might differ from what would be expected based on its skeletal density due to a de fluidized hood of bed material that appears on top of the object, as stated by Nguyen and Grace (1978) and Rees et al. (2005).

In a previous work (Soria Verdugo et al., 2011) the motion of a neutrally buoyant object within a $2 \mathrm{D}$ bed was analyzed. The object was found to describe cycles, moving from the surface of the bed to a certain depth and back to the surface by the action of several passing bubbles, as explained by Rios et al. (1986). The cycles described by the object were characterized in terms of the maximum attained depth and the number of jumps needed for the object to go back to the surface of the bed. The distribution of the maximum attained depth was found to be parabolic, whereas the distribution of the number of jumps showed an exponential decay.

In this work, the experimental procedure described in Soria Verdugo et al. (2011) has been applied to a variety of objects with different densities and sizes. The capability of the object to circulate in the bed is discussed and the effect of buoyant forces on the object sinking and rising processes is analyzed. The study was extended to fluidized beds with different dense phase diameter and bed height.

\section{Experimental setup}

The experiments were carried out in a $2 \mathrm{D}$ facility. The bed was set in a column with a width, $W$, of $0.5 \mathrm{~m}$, a height, $H$, of $2 \mathrm{~m}$ and a thickness, $T$, of $0.01 \mathrm{~m}$. The fixed bed height, $h_{b}$, was $0.5 \mathrm{~m}$, corresponding to a bed aspect ratio of 1 . The bed material was glass spheres (ballotini particles) with a skeletal density about $2500 \mathrm{~kg} / \mathrm{m}^{3}$ and a $600800 \mu \mathrm{m}$ of diameter, $d_{p}$, type B according to Geldart's (1973) classification. The bulk density of the fixed bed, $\rho_{b}$, was measured to be $1560 \mathrm{~kg} / \mathrm{m}^{3}$. The value of the minimum fluidization velocity, $U_{m f}$, was found to be $0.32 \mathrm{~m} / \mathrm{s}$ by means of pressure fluctuation analysis (using piezoelectric transducers of type Kistler 5015). A standard camera (Nikon D80 10.6 Mpx) was used to capture the object motion. The measurements of object
Table 1

Characteristics of the objects used in the experiments.

\begin{tabular}{lcl}
\hline Object & Density ratio $\left(\left(\rho_{b}-\rho_{o}\right) / \rho_{b}\right)$ & Shape ratio $\left(L_{O} / D_{O}\right)$ \\
\hline $\boldsymbol{F 1}$ & 0.66 & 3 \\
$\boldsymbol{F} 2$ & 0.21 & 3 \\
$\boldsymbol{N}$ & 0.03 & 3 \\
$\boldsymbol{J}$ & -0.09 & 3 \\
$\boldsymbol{L N}$ & 0.03 & 5 \\
$\boldsymbol{S N}$ & 0.03 & 1 \\
\hline
\end{tabular}

motion (to obtain both position and velocity) were carried out in complete darkness. The objects were coated with strontium aluminate to emit green light. Further details of the experimental setup can be found in Soria Verdugo et al. (2011).

The objects studied were in all cases of cylindrical shape, with a diameter, $D_{O}$, of $6.4 \mathrm{~mm}$, while their densities and lengths were varied in a broad range. The characteristics of the different objects are given in Table 1.

These six objects represented four different density ratios (bed object density difference over the bulk density of the fixed bed) and three different aspect ratios (length over diameter of the cylinder). Object $N$ represented a neutrally buoyant object, but it will behave in a slightly flotsam manner due to its density ratio. This object was analyzed in detail in Soria Verdugo et al. (2011) and it is given here as a quasi neutral reference in terms of buoyancy effects. Objects $F 1$ and $F 2$ had densities significantly below that of the bed and showed flotsam behavior. Object $J$ had a larger density than the bed density and showed jetsam behavior. Those four objects had the same aspect ratio. Objects $L N$ and $S N$ were, respectively, larger and smaller than object $N$, and with the same density and diameter. The dimensionless gas velocity was varied during the experiments between 1.5 and 3. At low fluidization velocities, some objects (particularly objects $J$ and $F 1$ ) did not circulate but settled over the distributor (object $J$ ) or remained on the bed surface (object $F 1$ ).

Finally, some experiments were performed to study the effect of bed height and bed material. In the first case, a shallower bed was used; with 0.75 bed aspect ratio (nominal case was 1 ). In the second case, the bed material was ballotini particles with a diameter in the range $400600 \mu \mathrm{m}$ (smaller than the nominal case), and with the same skeletal density. Those particles still correspond to Geldart's (1973) group B.

\section{Results}

The circulation time of an object immersed in a bubbling fluidized bed varies for each object cycle as a function of four main parameters: the mean sinking and rising velocities along the cycle, the maximum depth attained by the object and the number of jumps needed to get back to the surface of the bed. The distribution of the two last parameters will define the distribution of the circulation times of an object in a BFB. In Soria Verdugo et al. (2011), the distributions were obtained for the neutrally buoyant object, $N$. In this work, the effect of the object character istics and the bed parameters on such distributions is analyzed.

Fig. 1 shows the results obtained in Soria Verdugo et al. (2011) for the neutrally buoyant object, $N$, and compares them with those for a flotsam object (Object F1). Fig. 1(a) shows the relative frequency to find cycles with a determined number of jumps and Fig. 1(b) the relative frequency to find cycles that reach a determined maximum depth. The results were obtained for a dimensionless gas velocity of $U / U_{m f}=3$. The fitting curves for the results of the neutrally buoyant object are also depicted in Fig. 1, taken from Soria Verdugo et al. (2011). The distribution of the 
a

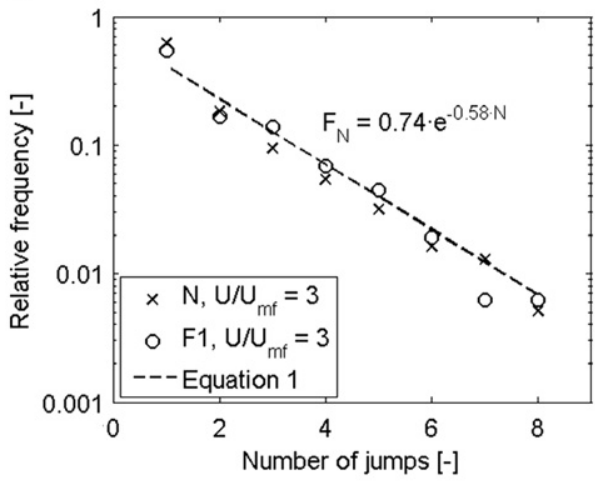

b

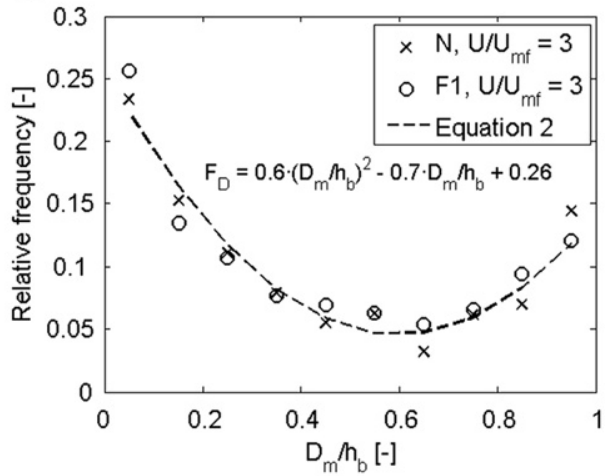

Fig. 1. Relative frequency (a) to find cycles with a determined number of jumps, and (b) to find cycles that reach a determined maximum depth.

a

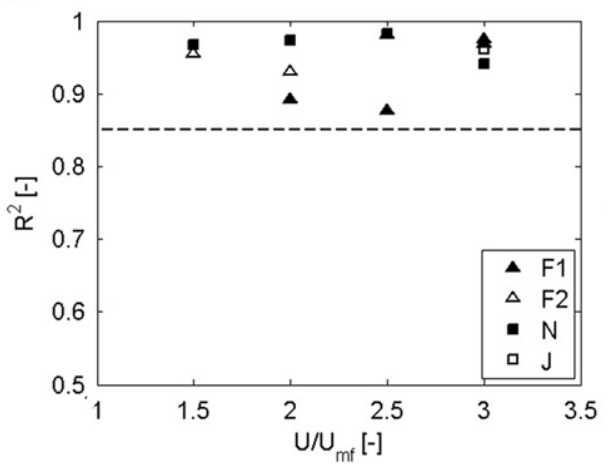

b

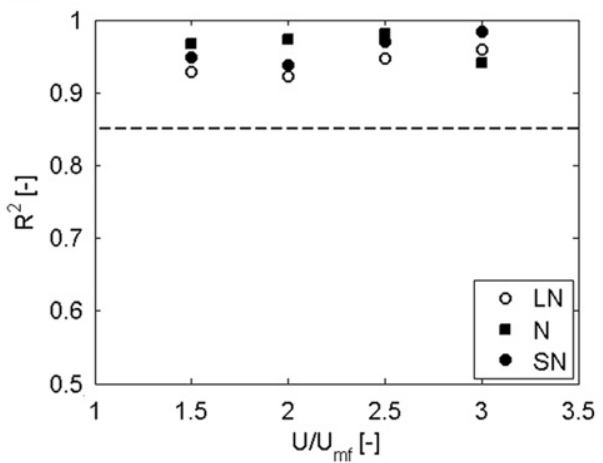

Fig. 2. $R^{2}$ coefficient, comparing the experimental data with Eq. (1), for the number of jumps distributions and for objects of different densities (a) and sizes (b).

number of jumps follows a negative exponential distribution, and the distribution of the maximum attained depths is parabolic. These distributions are shown in Eqs. (1) and (2), respectively.

$F_{N}=0.74 e^{0.58 N}$

$F_{D}=0.6\left(D_{m} / h_{b}\right)^{2} \quad 0.7\left(D_{m} / h_{b}\right)+0.26$

The experimental results are quite similar for both objects and the fittings obtained for object $N$ seem to represent accurately both distributions, independently of the object density.

The motion of the rest of objects was analyzed at different air velocities and the results are presented in Figs. 25.

The experimental data for the number of jumps showed in all cases surprisingly similar tendencies to the results already shown in Fig. 1(a). On the basis of such a similarity, no independent fittings were presented for each dataset, but they were directly compared with the fitting obtained for the neutrally buoyant object, presented in Eq. (1). An $R^{2}$ adjustment coefficient was then calculated between each dataset and Eq. (1) to characterize the overall validity of the equation. The $R^{2}$ coefficient was calculated by the least squares method, and, being the distribution expo nential, the coefficient is calculated over the logarithmic data.

Fig. 2 shows the $R^{2}$ coefficients of the number of jumps distributions, for objects with different densities (a) and sizes (b), and for a range of air velocities.

In all cases, the $R^{2}$ coefficient with Eq. (1) was higher than 0.85 , a value that can still be considered adequate. Therefore, Eq. (1) accurately represents the global behavior concerning the number of jumps, which can be said to be independent of the object density and size and of the air velocity, for the ranges studied.

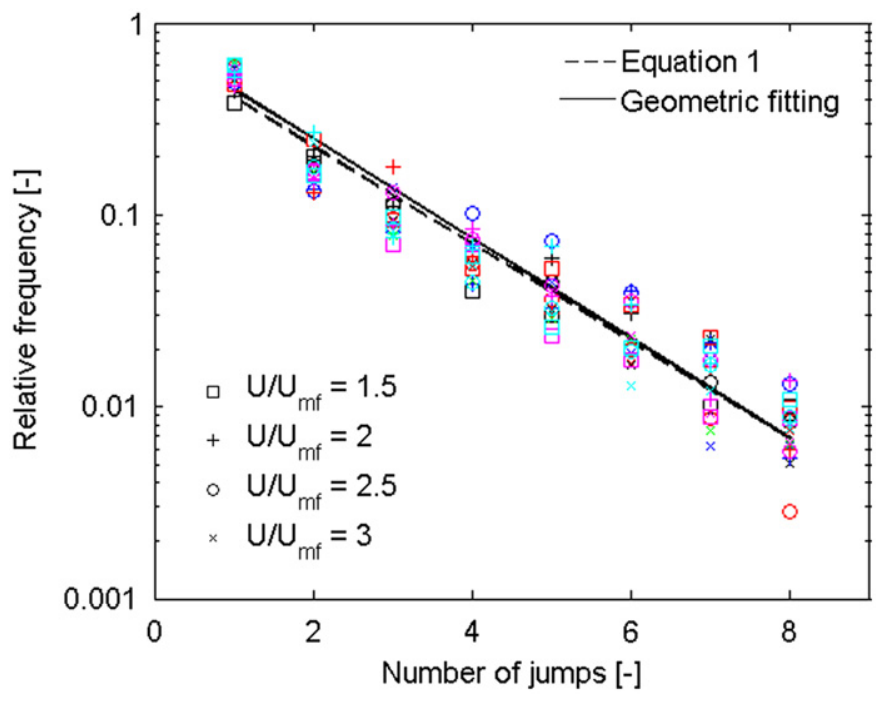

Fig. 3. Relative frequency to find cycles with a determined number of jumps for all the tests. Black color object $N$, blue color object $F 1$, red color object $F 2$, green color object J, magenta color object SN and cyan color object LN. (For interpretation of the references to color in this figure legend, the reader is referred to the web version of this article.)

The experimental data corresponding to all the tests are plotted in Fig. 3 along with Eq. (1). A small scatter of the data can be observed in the figure, but the similar behavior established in Fig. 2 is evident. 
a

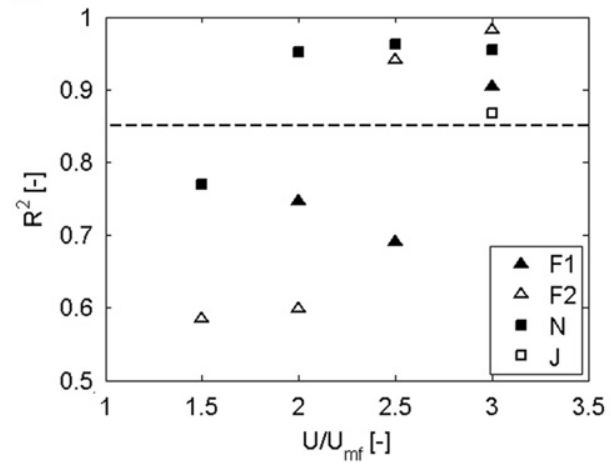

b

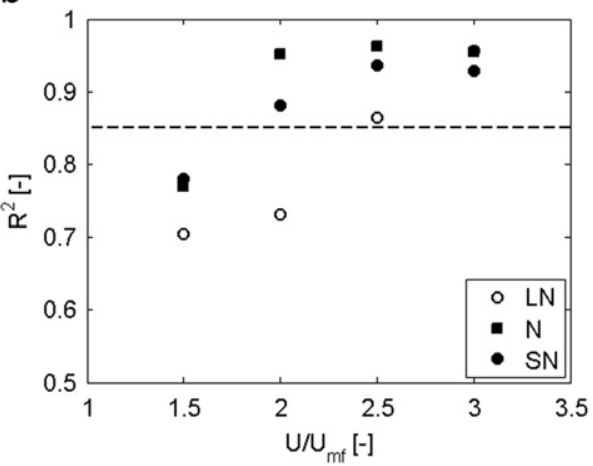

Fig. 4. $R^{2}$ coefficient, comparing the experimental data with Eq. (2), for the maximum attained depth and for objects of different densities (a) and sizes (b).

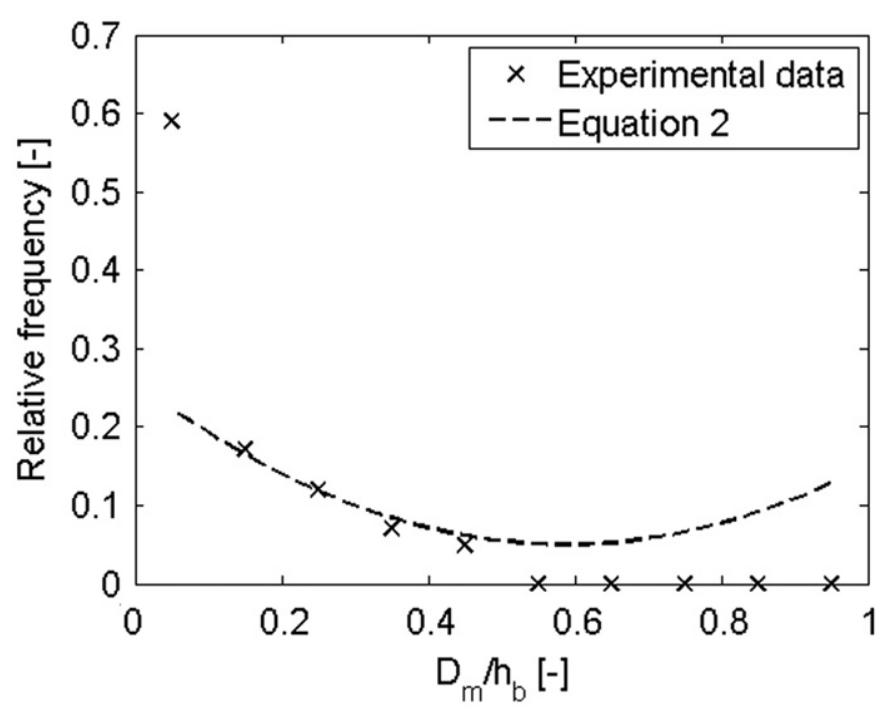

Fig. 5. Relative frequency to reach a determined depth. Flotsam object F2, operating at $U / U_{m f} 1.5$.

The exponential distribution being used up to now in our calculations has a drawback in properly representing the data. It is a continuous distribution while the number of jumps is a discrete variable $(N=1,2,3 \ldots)$. Therefore, the exponential fitting of Eq. (1) should be transformed into a discrete distribution. The discrete analog of the exponential distribution is the geometric distribution. Its probability density function is given by Eq. (3).

$P(N)=p(1 p)^{N-1}$ for $N=1,2,3 \ldots$

The geometric distribution that fits the data of object $N$ is also plotted in Fig. 3, showing minor differences with Eq. (1). The value of $p$ in the fitting $(p=0.45)$ describes the average probability that an object has, when it is about to take a jump, to be lifted directly to the bed surface. The value $1 p,(1 p=0.55)$, describes the average probability of an object to, once started to rise, detach from the bubble or its drift and begin a new sinking process. No object, at any position and time, was ever observed to remain static, but it was either following a rising or a sinking process.

Note that the probability that an object has, when it is about to take a jump, to be lifted directly to the bed surface is independent of its previous history of jumps. This is derived from the fact that the distribution of the number of jumps is geometrical (or exponential). Such distributions represent memory less pro cesses. The process of having to perform a new jump should then represent a memory less process, where the probability of a new jump is independent of the number of previous jumps.

On the other hand, this is an average probability. Only this average remains constant. The experiments showed that, when the averaging was performed separating the different depths at which the object begins to rise, the average probabilities varied. Evidently enough, probabilities to reach the bed surface were higher for lower depths. This distribution for varying depths may change. Also, the probability of the object to begin its rise at a certain depth varies with depth and may vary for the different experimental conditions. But the global average remains constant as established in Figs. 2 and 3.

The main result of this analysis, shown in Figs. 2 and 3, is that the average probabilities for the object rising path (45\% of reach ing the surface and 55\% of detaching and start a new sinking process) are independent of object properties such as density and size and of the excess gas velocity. At the end of this section, the effect of bed height and bed material on these average probabil ities will be also discussed.

The distribution of probability of the attained depth was also studied in detail. As was the case in the study of the number of jumps, some coherence is observed in the results and an $R^{2}$ coefficient was calculated between the different datasets and the parabolic fitting shown in Eq. (2) (obtained for the neutrally buoyant object) for the maximum attained depth. The results for all the tests are reported in Fig. 4. As done previously, the validity of the fitting was accepted for $R^{2}$ coefficients larger than 0.85 . All objects showed a proper adjustment to Eq. (2) for the highest dimensionless air velocity tested $\left(U / U_{m f}=3\right)$. For lower gas velo cities, density and size effects became important and the dis tribution changed, first $\left(U / U_{m f}=22.5\right)$ for lower densities and larger objects and finally $\left(U / U_{m f}=1.5\right)$ for all objects. The dis tribution obtained for the jetsam object was symmetrical to those of the flotsam objects. Compared to the symmetrical of $F_{D}$, it showed a proper adjustment $\left(R^{2}=0.87\right)$. The results for the jetsam object are only presented for $U / U_{m f}=3$. For lower air velocities, the object seldom reached the bed surface (less than 5 cycles per hour). No proper circulation can then be stated and thus these data points are omitted.

All the tests where the results differ considerably from Eq. (2) $\left(R^{2}<0.85\right.$ in Fig. 4) showed a similar tendency. Although the distributions for medium or small depths still followed the equation, this was not the case for large depths and also, what is less obvious, for positions very near to the bed surface. This is shown in Fig. 5, where the results for the flotsam object $F 2$, operating at $U / U_{m f}=1.5$, are presented.

This similar evolution for all the cases that are not matched by Eq. (2) can be explained by an inadequate circulation. Direct 
observation showed that most of the time the objects were retained in the surroundings of the bed surface and seldom descended to large depths. This produced the observed transfer of relative frequencies from larger to lower depths.

This inability to access to large depths should be associated to the incidence of buoyant forces during the sinking path of the object. Note that the results that reproduce the tendency shown in Fig. 5 and thus mismatch Eq. (2) in the two graphs of Fig. 4 are those obtained for low air velocities and lighter or larger objects. Reducing the object density would produce a higher buoyant force and therefore smaller sinking velocities. This will prevent the object from sinking deep in the bed and thus the probability to attain large depths will diminish. The effect of the size seems to act in quite a similar way, with larger objects experimenting larger buoyant forces. The strong air velocity effect can be considered to be due to the increase of the fluidization intensity (Otshitani et al., 2004). A large fluidization intensity reduced the effect of buoyant forces, allowing the object to move throughout the whole bed. As a general conclusion, it can be stated that Eq. (2) can be considered to accurately represent the distribution for all the objects when their ability to move throughout the bed is ascertained.

The probability density function for the maximum attained depth can be obtained from Eq. (2). The attained depth is a continuous variable in contrast to the number of jumps. There fore, its probability density function is obtained by normalizing Eq. (2), giving Eq. (4).

$P_{D}=5.45\left(\frac{D_{m}}{h_{b}}\right)^{2} \quad 6.36\left(\frac{D_{m}}{h_{b}}\right)+2.36$

The bed capability of maintaining the object in circulation throughout the whole bed, as a function of object and bed parameters, is a key point. For a proper understanding, it has been analyzed using a different approach. The object position was recorded during all the experiments and a parameter, $F_{u z}$, was defined and calculated as the probability to find the object in the upper half of the bed. This parameter would be close to $50 \%$ for an object that circulates evenly throughout the bed, and can be indistinctly found at the higher or at the lower part of the bed. This would be the case for an adequate bed dynamics and for neutrally buoyant objects. On the other hand, its value would approach $100 \%$ for highly flotsam objects and poor dynamics, which maintain the objects in the surrounding of the bed surface. In such cases, the object seldom appears at the bottom zone. Finally, its value would be close to $0 \%$ for highly jetsam objects and poor bed dynamics, in which case the object sinks and remains over the distributor and rarely rises to the surface.

The results of the parameter $F_{u z}$ for the different objects and excess gas velocities are shown in Fig. 6 . These results can be compared with those shown in Fig. 4, since a low $R^{2}$ coefficient in those graphs was linked to poor circulation. The two dashed lines in the graphs of Fig. 6 are obtained from this comparison. They represent a $\pm 15 \%$ interval around $F_{u z}=50 \%$. The data outside such an interval showed low $R^{2}$ coefficients as a result of poor circulation, while the data inside the interval correspond to $R^{2}$ coefficients higher than 0.85 . The results are, therefore, consistent between the two different approaches.

All the objects tested had a proper circulation throughout the bed for the highest gas velocity tested $\left(U / U_{m f}=3\right)$. The neutrally buoyant object, $N$, had a proper circulation for a range of dimensionless gas velocities, but it drifts from the $50 \%$ zone for decreasing gas velocities and reaches the limit for $U / U_{m f}=1.5$. Reducing the density ratio, the case of objects $F 2$ and $F 1$, produced a steeper effect of the gas velocity. The object $F 2$ circulated properly throughout the bed for dimensionless gas velocities higher than 2.5, but for lower values ( 1.5 and 2 ) buoyancy effects became important and the probability to find the object in the upper half of the bed was higher. Operating with lighter objects, such as $F 1$, buoyant forces precluded the proper circulation of the object for dimensionless gas velocities as high as 2.5 , but the object circulated throughout the whole bed for $U / U_{m f}=3$. The jetsam object, $J$, was capable of circulate within the bed for the highest dimensionless air velocity tested $\left(U / U_{m f}=3\right)$, but when reducing the air velocity it sank deep in the bed due to buoyant forces. The value of $F_{u z}$ for the jetsam object became 0 when operating at 1.5 and 2 times $U_{m f}$, since the object just rested over the distributor in such cases. In all cases, an increase of the air velocity and the linked increase of the fluidization intensity improved the circulation of objects throughout the bed, reducing the effect of buoyant forces.

A higher effect of density differences between object and bed is apparent for the jetsam object (density ratio 0.09) in comparison with the flotsam objects (density ratios 0.21 and 0.66). This effect seems to be a consequence of the opposite or identical directions between buoyant forces and dense phase motion. The neutrally buoyant object has shown a slight flotsam behavior in all the results presented.

The effect of object size, presented in Fig. 6(b) for the three objects $N$ tested, shows some coherent evolution, although the size effect seems slightly smaller than the density effect. The three objects $N$ are slightly flotsam, as established in Fig. 6(a), and the larger the object the more flotsam it became. A generalization of the size effect to jetsam objects is rather tricky. This might mean that larger sizes strength the flotsam/jetsam behavior of the particle, or that large sizes increase object flotation. Further work is needed to establish this point.

A ratio between the time that an object with a given density and size is on the bed surface or the splash region and that which a

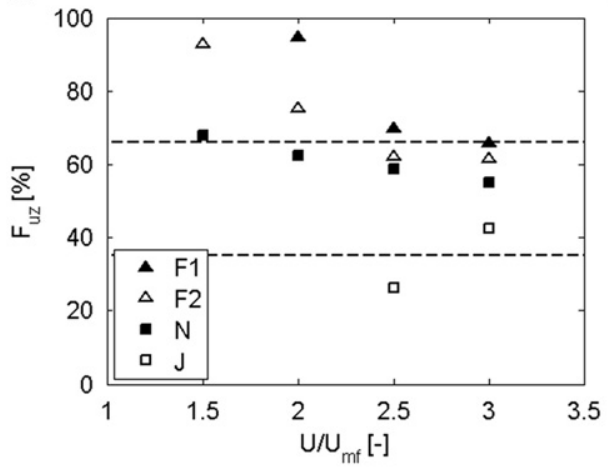

b

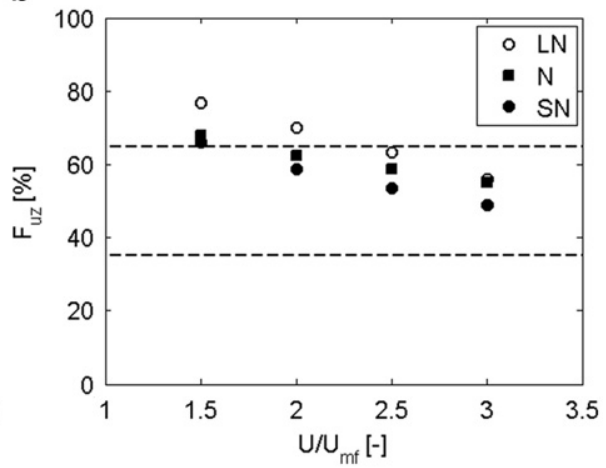

Fig. 6. Probability to find the object in the upper half of the bed for (a) different object densities and (b) sizes. 
a

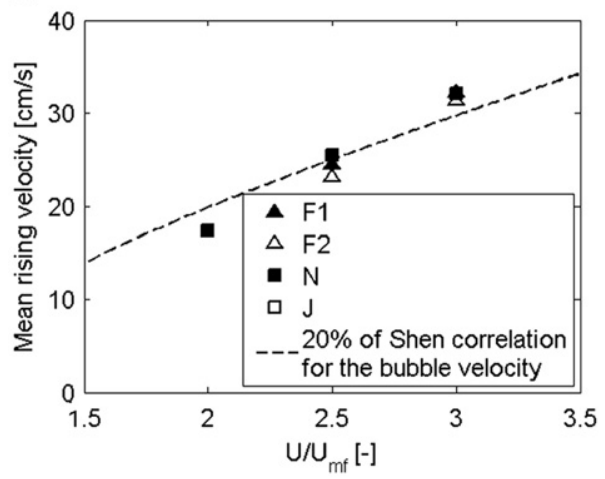

b

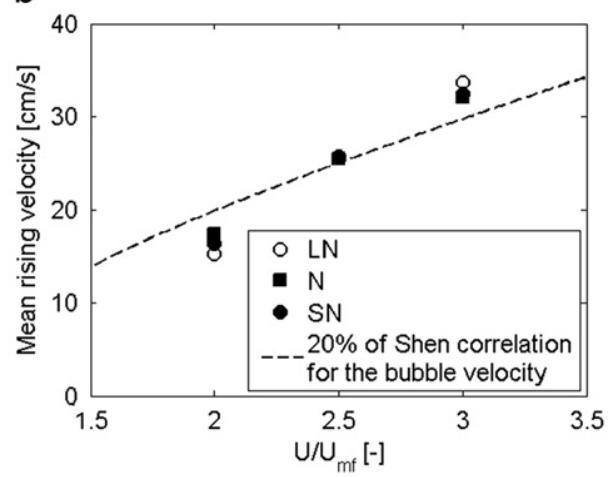

Fig. 7. Mean rising velocity of objects with different densities (a) and sizes (b).

is spent inside the bed can also be obtained from this experi ments. For the object $N$ it is around $7 \%$. When varying object density and size, the ratio slightly increases for more buoyant objects. This increase is clearer when increasing $U / U_{m f}$. The density and size effect can be attributed to buoyant forces, while the effect of the gas velocity can be linked to an increase in the bubble capability of ejecting objects.

Finally, the object velocity inside the bed was determined and compared to well established correlations for bubbles and dense phase motion. The mean sinking and rising velocities were calculated using a procedure described in Soria Verdugo et al. (2011). The position of the object is obtained and sampled using a sampling frequency of $2 \mathrm{~Hz}$ (to avoid the presence of vibration velocities in the averaging); then, the two averages are obtained, separating upwards and downwards velocities. The mean rising velocity of the object can be observed in Fig. 7 for all experimental conditions and objects. The results show similar mean rising velocities for all the objects, and a quasi linear behavior with gas velocity. Objects were unable to circulate properly throughout the bed for $U / U_{m f}=1.5$ so those results are missing. The results are compared with the mean velocity of bubbles, calculated using the correlation of Shen et al. (2004) for the bubble diameter, $D_{B}$, and the correlation of Davidson and Harrison (1963) for the bubble velocity, $U_{B}$. These correlations are:

$\left.D_{B}=\left[\left(\frac{8\left(2^{3 / 4} 1\right.}{\lambda}\right)\left(\begin{array}{ll}U & U_{m f}\end{array}\right)\left(h+\frac{\lambda}{\pi\left(2^{3 / 4} 1\right.}\right) \frac{A_{0}}{T}\right)\right]^{2 / 3} g^{-1 / 3}$

$U_{B}=U \quad U_{m f}+\phi \sqrt{ } g D_{B}$

Several authors (Rios et al., 1986; Nienow et al., 1978; Lim and Agarwal, 1994; Rees et al., 2005) have studied the mean rising velocity of objects in fluidized beds, but their results present a wide dispersion. The ratio of the mean rising velocity of the object and the mean bubble velocity along the bed ranged between $10 \%$ and $30 \%$ depending on the study. Our results show a reasonable agreement for a $20 \%$ ratio.

The low variability of the object rising velocity with the density and size suggests that the bubbles capability of raising them is not influenced by these parameters.

The mean sinking velocities for all objects and excess gas velocities are shown in Fig. 8. A certain effect of buoyant forces can be established. The sinking velocities of heavier objects are larger. A similar effect is observed for smaller objects of the slightly buoyant type $N$. This suggests the existence of a super imposed buoyant velocity that is a function of these two para meters: the density difference between the object and the surrounding bed and the object length. A third parameter of importance here, although unstudied in this work, would be the object shape. A comparison with the dense phase velocity is also given, using the correlation of Kunii and Levenspiel (1991). It shows a reasonable agreement with the data for object $N$. The correlation is given in Eq. (6).

$v_{d p}=\frac{f_{w} \delta U_{B}}{1 \delta f_{w} \delta}$

The experimental results of Figs. 18 gave a coherent char acterization of the effect of buoyant forces in the motion of objects immersed in a bed, for the range of parameters presented. The rising process is connected to the bubble motion and the rising velocity dependence on gas velocity follows well estab lished correlations for bubble velocity. On the other hand, buoy ant effects seem inexistent concerning the rising path: the number of jumps and the rising velocity are not much affected by object density or size. Nevertheless, the sinking process and parameters related show a clear buoyant effect. This is evident when looking at Figs. 6 and 8, and for the cases in Fig. 4 that do not follow the otherwise general parabolic fit of Eq. (2). Increasing the gas velocity produces a more vigorous fluidization and enables the object to circulate throughout the bed, diminishing the effect of buoyant forces. Furthermore, Fig. 8 shows that, while the mean sinking velocity clearly increased with the gas velocity, the differences in mean sinking velocity between flotsam and jetsam objects and between larger and smaller objects did not overcome large variations. Therefore, the ratio between buoyant velocities and neutrally buoyant sinking velocities decreases for increasing gas velocities and the buoyant effect becomes less significant.

The results previously shown for the probability distributions of the number of jumps and the attained depth indicate that the distributions remain almost identical for different objects and gas velocities. In order to test the validity and range of those expressions, the effects of bed height and bed material were also tested.

Previous experiments were carried out in a bed with a bed aspect ratio (height over width) of 1 . Fig. 9 shows the results for a lower bed height $\left(h_{b} / W=0.75\right)$. Note that the abscissas in Fig. 9(b) denote different depths for different bed heights.

The correlations of Eqs. (1) and (2) seem to perfectly fit the experimental data in Fig. 9. Therefore, the same correlation can be applied to beds with different aspect ratios.

Finally, the effect of the bed material was tested. Previous experiments were carried out in a bed conformed by ballotini particles with $600800 \mu \mathrm{m}$ diameter range. In the experiments shown in Fig. 10, the motion of the neutrally buoyant object, $N$, was studied in a bed of ballotini particles with $400600 \mu \mathrm{m}$ diameter range. The skeletal density of the particles remained constant, and in all cases they correspond to Geldart's (1973) group B. 
a

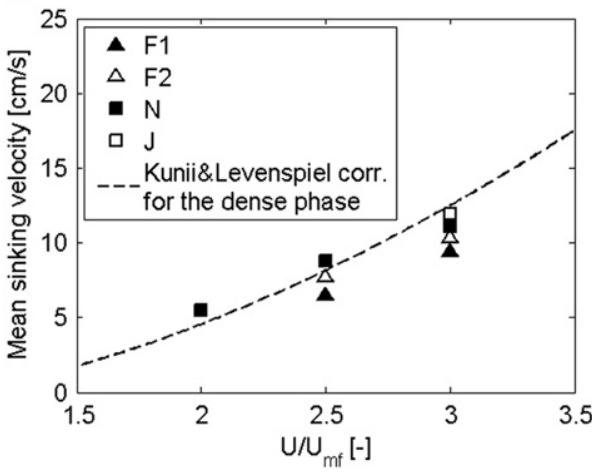

b

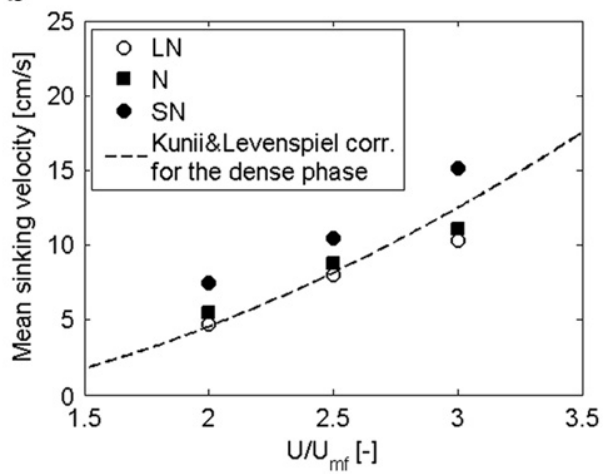

Fig. 8. Mean sinking velocity of objects with different densities (a) and sizes (b).

a

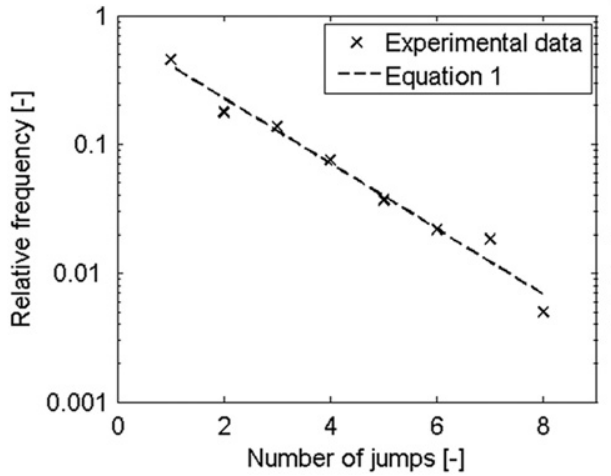

b

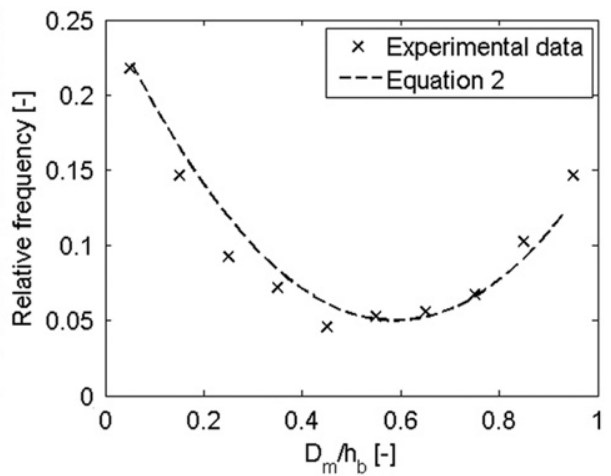

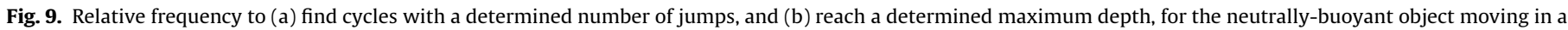
bed with a bed aspect ratio $h_{b} / W \quad 0.75$ operating at $U / U_{m f} 3$.

a

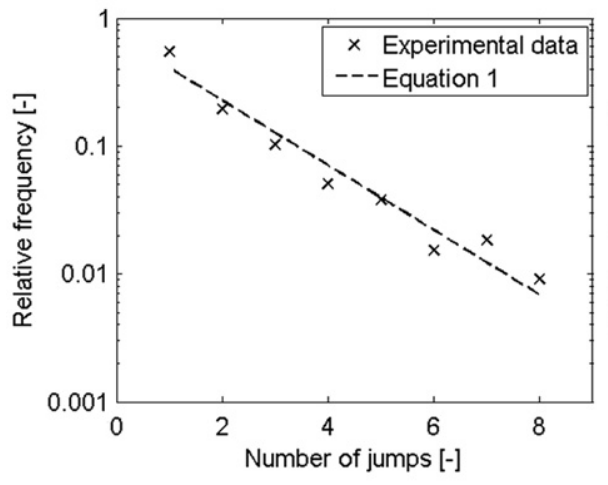

b

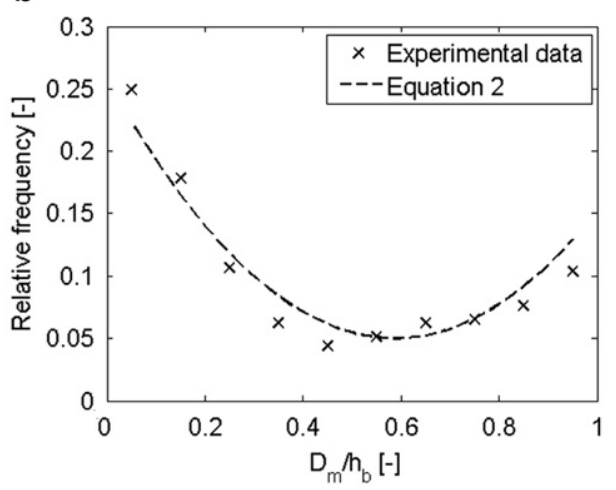

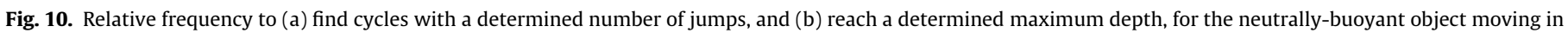
a bed with dense material of a diameter $400-600 \mu \mathrm{m}$ operating at $U / U_{m f} 3$.

Once again, no important differences can be observed between the experimental data in Figs. 9 and 10 and the correlations obtained for the previous experiments. Therefore, the probability distributions given in Eqs. (1) and (2) remain also independent of the bed material diameter for the ranges studied in this work. Those expressions are thus universal for the range of our experiments. Therefore, this procedure can be used to obtain a simple and reliable estimate of the circulation time of the different objects in different bed configurations, as described in Soria Verdugo et al. (2011).

The results of this work show the influence of buoyancy in the sinking and rising motion of a particle immersed in a bubbling fluidized bed. The sinking motion is shown to be affected by buoyancy, while the rising behavior is quite independent of buoyancy effects, in two main aspects of the object motion: its mean rising velocity and the number of jumps needed to reach the bed surface (the number of bubbles required to raise it to the surface). Some effort has been made to establish the indepen dence of this behavior of the bed configuration and the relative importance of buoyant forces. Nevertheless, the conclusions should not be regarded as directly applicable to any bed, and in particular to industrial $3 \mathrm{D}$ beds. First, this is a $2 \mathrm{D}$ study, and the wall effect is significant. The differences in bubble and dense phase motion between $2 \mathrm{D}$ and $3 \mathrm{D}$ beds have long been 
established. The shear layer in the dense phase may not have a strong effect in a far larger object, but the object wall shear may affect the results. Finally, in a $3 \mathrm{D}$ bed an object is able to rotate in a perpendicular plane to its rising motion, and this may produce significant differences in the attachment and detachment pro cesses between object and bubble. Other limitation of this study is the gas velocity range. $U / U_{m f}=3$ is quite a maximum in a $0.5 \mathrm{~m}$ bed, due to slugging effects, but far larger velocities can be obtained in industrial beds, even bubbling beds. The results may be extrapolated for $U / U_{m f}$ higher than 3 but close to this value, but the extrapolation should not be strained, as different phenomena are prone to appear.

\section{Conclusions}

The effect of buoyant forces on the motion of a large cylind rical object immersed in a $2 \mathrm{D}$ bubbling fluidized bed was experimentally studied using digital image analysis. The results show that a proper circulation and axial homogeneity is affected by object density, size and gas velocity. For slightly buoyant particles, increasing the object size has a similar but less intense effect than decreasing object density.

The results show that the probability distribution of the number of jumps is independent of density and size of the object and gas velocity and also of other bed parameters such as the bed height and the particle size distribution of the bed material. In all cases, the probabilities followed a geometric distribution for the number of jumps of the form $P_{N}=0.45 \cdot(0.55)^{N}{ }^{1}$. The value 0.45 describes the average probability of an object to, once started to rise, be lifted directly to the bed surface. It is found to remain constant for all experimental conditions analyzed in this work, including gas velocity changes and varying object density and size.

A similar result is found for the probability distribution of the maximum attained depth, which is found to follow the same parabolic law in all cases. Nevertheless, this is only valid when a proper circulation throughout the bed is ensured.

Rising and sinking velocities are highly dependent on gas velocity, and can be related to well known models of bubble and dense phase velocities. Nevertheless, rising velocities are practically unaffected by object density or size, while sinking velocities show a low dependence on density and a steeper one on size.

As a main conclusion, buoyant forces are found to be relevant during the sinking process, but no significant effect is observed during the rising path, for the ranges of variation studied in this work. The mean rising velocity of objects and the ability of bubbles to rise objects is unaffected by object density and size. On the other hand, the sinking motion varies with the incidence of buoyant forces, showing axial circulation non homogeneity, mean sinking velocity variations and restrictions of flotsam or jetsam objects to remain in the upper or bottom region, respec tively. The effect of buoyant forces on the object motion can be reduced by increasing the gas velocity.

\section{Nomenclature}

$A_{O} \quad$ area of the distributor per number of orifices

$d_{p} \quad$ dense phase particles diameter

$D_{O} \quad$ object diameter

$D_{B} \quad$ bubble diameter

$D_{m} \quad$ maximum attained depth

$f_{w} \quad$ bubble wake fraction

$F_{D} \quad$ relative frequency to attain a certain depth
$F_{N} \quad$ relative frequency of a certain number of jumps

$F_{u z} \quad$ relative frequency to find the object at the upper half of the bed

g gravity

$h \quad$ height over the distributor

$H \quad$ vessel height

$h_{b} \quad$ fixed bed height

$L_{O} \quad$ object length

$N$ number of jumps needed for the object to rise to the surface of the bed

$p \quad$ average probability of a rising object to be lifted directly to the bed surface

$P_{D} \quad$ probability density function to attain a certain depth

$P_{N} \quad$ probability density function of the number of jumps

$R^{2} \quad$ determination coefficient

$T \quad$ bed thickness

$U \quad$ superficial gas velocity

$U_{B} \quad$ bubble velocity

$U_{m f} \quad$ minimum fluidization velocity

$v_{d p} \quad$ downward velocity of the dense phase

$W \quad$ bed width

$\delta \quad$ bubble fraction in the bed

$\lambda \quad$ constant determined experimentally

$\phi \quad$ constant determined experimentally

$\rho_{b} \quad$ packed bed density

$\rho_{o} \quad$ object density

\section{Acknowledgments}

This work has been partially supported by the National Energy Program of the Spanish Department of Science and Education (ENE2006 01401), the Spanish Government (DPI2009 10518 MICINN) and the Madrid Community (CCG07 uc3m/amb 3412 and CCG08 uc3m/amb 4227).

\section{References}

Bokkers, G.A., van Sint Annaland, M., Kuipers, J.A.M., 2004. Mixing and segregation in a bidisperse gas-solid fluidised bed: a numerical and experimental study. Powder Technology 140 (3), 176-186.

Davidson, J.F., Harrison, D., 1963. Fluidised Particles, 1st ed. Cambridge University Press, Cambridge.

Du, B., Wei, F., Warsito, W., 2002. Gas and solids mixing in a turbulent fluidized bed. AIChE Journal 48 (9), 1896-1909.

Geldart, D., 1973. Types of gas fluidization. Powder Technology 7, 285-292.

Grassler, T., Wirth, K.-E., 2000. X-ray computer tomography-potential and limitation for the measurement of local solids distribution in circulating fluidized beds. Chemical Engineering Journal 77 (1-2), 65-72.

Ibsen, C.H., Solberg, T., Hjertager, B.H., Johnsson, F., 2002. Laser Doppler anemometry measurements in a circulating fluidized bed of metal particles. Experimental Thermal and Fluid Science 26 (6-7), 851-859.

Kunii, D., Levenspiel, O., 1991. Fluidization Engineering, 2nd ed. ButterworthHeinemann, Boston.

Lim, K.S., Agarwal, P.K., 1994. Circulatory motion of a large and lighter sphere in a bubbling fluidized bed of smaller and heavier particles. Chemical Engineering Science 49 (3), 421-424.

Nguyen, T.H., Grace, J.R., 1978. Forces on objects immersed in fluidized beds Powder Technology 19, 255-264.

Nienow, A.W., Rowe, P.N., Chiba, T., 1978. Mixing and segregation of a small portion of large particles in gas fluidized beds of considerably smaller ones. AIChE Symposium Series 74, 45-53.

Otshitani, J., Keiko, O., Makoto, I., Zennosuke, T., 2004. Effect of particle fluidization intensity on floating and sinking of objects in a gas-solid fluidized bed. Advanced Powder Technology 15 (2), 201-213.

Pallarès, D., Johnsson, F., 2006. A novel technique for particle tracking in cold 2-dimensional fluidized beds-Simulating fuel dispersion. Chemical Engineering Science 61, 2710-2720.

Rees, A.C., Davidson, J.F., Hayhurst, A.N., 2005. The rise of a buoyant sphere in a gas-fluidized bed. Chemical Engineering Science 60, 1143-1153.

Rios, G.M., Dang Tran, K., Masson, H., 1986. Free object motion in a gas fluidized bed. Chemical Engineering Communications 47, 247-272. 
Shen, L., Johnsson, F., Leckner, B., 2004. Digital image analysis of hydrodynamics two-dimensional bubbling fluidized beds. Chemical Engineering Science 59, 2607-2617.

Soria-Verdugo, A., Garcia-Gutierrez, L.M., Sanchez-Delgado, S., Ruiz-Rivas, U. 2011. Circulation of an object immersed in a bubbling fluidized bed. Chemica Engineering Science 66, 78-87.
Stein, M., Ding, Y.L., Seville, J.P.K., Parker, D.J., 2000. Solids motion in bubbling gas fluidised beds. Chemical Engineering Science 55, 5291-5300.

Tanimoto, H., Chiba, S., Chiba, T., Kobayashi, H., 1981. Jetsam descent induced by a single bubble passage in three-dimensional gas-fluidized beds. Journal of Chemical Engineering of Japan 14 (4), 273-276. 